\title{
A MATHEMATICAL MODEL FOR FIBRO-PROLIFERATIVE WOUND HEALING DISORDERS
}

\author{
LUKE OLSEN, ${ }^{*}$ JONATHAN A. SHERRATT, $\dagger$ and \\ PHILIP K. MAINI* \\ ${ }^{*}$ Centre for Mathematical Biology, \\ Mathematical Institute, \\ 24-29 St Giles', \\ Oxford, OX1 3LB, United Kingdom \\ (E.mail: olsen@vax.ox.ac.uk) \\ $†$ Nonlinear Systems Laboratory, \\ Mathematics Institute, \\ University of Warwick, \\ Coventry, CV4 7AL, United Kingdom
}

The normal process of dermal wound healing fails in some cases, due to fibro-proliferative disorders such as keloid and hypertrophic scars. These types of abnormal healing may be regarded as pathologically excessive responses to wounding in terms of fibroblastic cell profiles and their inflammatory growth-factor mediators. Biologically, these conditions are poorly understood and current medical treatments are thus unreliable.

In this paper, the authors apply an existing deterministic mathematical model for fibroplasia and wound contraction in adult mammalian dermis (Olsen et al., J. theor. Biol. 177, 113-128, 1995) to investigate key clinical problems concerning these healing disorders. A caricature model is proposed which retains the fundamental cellular and chemical components of the full model, in order to analyse the spatiotemporal dynamics of the initiation, progression, cessation and regression of fibro-contractive diseases in relation to normal healing. This model accounts for fibroblastic cell migration, proliferation and death and growth-factor diffusion, production by cells and tissue removal/decay.

Explicit results are obtained in terms of the model processes and parameters. The rate of cellular production of the chemical is shown to be critical to the development of a stable pathological state. Further, cessation and/or regression of the disease depend on appropriate spatiotemporally varying forms for this production rate, which can be understood in terms of the bistability of the normal dermal and pathological steady states - a central property of the model, which is evident from stability and bifurcation analyses.

The work predicts novel, biologically realistic and testable pathogenic and control mechanisms, the understanding of which will lead toward more effective strategies for clinical therapy of fibro-proliferative disorders.

1. Introduction. The normal healing response of full-thickness dermal excisional wounds may be considered within the context of three continu-

*Address correspondence to P. K. Maini. 
ous, overlapping temporal phases, termed "inflammation," "proliferation" and "remodelling" (Clark, 1988; Kirsner and Eaglstein, 1993). During the proliferative phase, wound closure is usually achieved by a combination of wound contraction and new tissue formation (Rudolph et al., 1992; Skalli and Gabbiani, 1988), with the proportions of these contributions dependent upon species, age and the location, extent and geometry of the wound.

The complex, interregulatory cellular events whereby fibroblasts proliferate into the wound site, secrete growth factors and extracellular matrix proteins, differentiate into myofibroblasts and effect wound contraction are collectively termed "fibroplasia," a constitutive feature of the proliferative phase of normal healing (Clark, 1993). The underlying mechanisms of these processes are increasingly well delineated and have attracted considerable investigation, both experimental (see Clark, 1993; Jennings and Hunt, 1992; Kirsner and Eaglstein, 1993; Mast, 1992; Rudolph et al., 1992 for thorough reviews) and theoretical (focusing primarily on the contractile effects of cell-matrix interactions during fibroplasia (Olsen et al., 1995; Tranquillo and Murray, 1992; Traqui et al., 1996)).

Abnormal regulation of fibroplasia and wound contraction, however, may result in one of a wide range of healing disorders (see Asmussen and Söllner, 1993; Skalli and Gabbiani, 1988 for overviews). For example, excessive dermal responses can lead to gross hypertrophic scars (in which the healed wound tissue becomes elevated above the plane of the skin) or keloids (in which the scar does not remain confined to the original wound margins), characterized by increased levels of cellularity (fibroblasts and myofibroblasts), collagen deposition and inflammatory growth factors (Ehrlich et al., 1994; Murray and Pinnell, 1992). In addition, active cell traction generates intense mechanical stresses, especially around the scar periphery, resulting in physical pain. Extensive scarring may also cause psychological trauma (Murray and Pinnell, 1992).

Detailed biological insight into the mechanisms of pathogenesis, progression, stabilization and regression of these "fibro-contractive diseases" is lacking, and existing clinical corrective methods are typically long-term, unpredictable, traumatic for the patient and prone to failure or recurrence. These include extensive surgical excision of affected tissues, full-thickness skin grafting and mechanical compression (Asmussen and Söllner, 1993; Boykin and Molnar, 1992; Murray and Pinnell, 1992; Rudolph et al., 1992).

Phenomenologically, fibro-contractive diseases commonly exhibit increased fibroplastic and retractile tissue responses in which myofibroblasts are ubiquitous cellular components (Gabbiani, 1992; Skalli and Gabbiani, 1988), but otherwise, the features of these pathologies are highly diverse (Murray, 1993; Skalli et al., 1989). Excessive disorganized extracellular matrix deposition (notably fibrillar collagen) and continual inflammatory 
responses are also characteristic of many fibro-contractive diseases (Ehrlich et al., 1994; Murray, 1993). Most of these pathologies stabilize with time, and some even partially or completely regress, but the determinants of this behaviour are unknown (Asmussen and Söllner, 1993; Murray and Pinnell, 1992).

Some fibro-contractive conditions arise from inflammatory settings, such as wound healing and soft tissue remodelling; these include severe burn scar contractures (with similar consequences to Dupuytren's disease), hypertrophic scar, keloids, liver cirrhosis, kidney and liver fibroses, regenerating tendon, cataract and fibrotic capsules surrounding tissuc implants (Boykin and Mollnar, 1992; Ehrlich et al., 1994; Murray and Pinnell, 1992; Schürch et al., 1990; Skalli and Gabbiani, 1988). Others, known as fibromatoses, are non-inflammatory, non-invasive (myo-)fibroplastic lesions; an example is the well-documented Dupuytren's contracture, a permanent condition affecting the upper palmar skin which consists of actively contractile cellular nodules and fibrous connective tissue cords (Flint, 1990; Rudolph and Vande Berg, 1991; Schürch et al., 1990), resulting in severe discomfort and loss of function of the affected hand. There are other proliferative tissue disorders with important similarities which do not arise from physical injury; an important class is those believed to be neoplastic responses, characterized by vigorous myofibroblastic proliferations in the stroma of invasive carcinomas (Skalli and Gabbiani, 1988).

In this paper, we focus on the inflammatory growth-factor-mediated fibro-proliferative responses associated with wound healing disorders. These are investigated within the framework of a mathematical model recently proposed for fibroplasia and wound contraction (Olsen et al., 1995) with the aim of an improved understanding of the pathogenic mechanisms of such conditions and their relationships to normal healing.

\section{Mathematical Modelling.}

2.1. The full model framework. We have recently proposed a continuum model for fibroplasia and wound contraction in adult mammalian dermis (Olsen et al., 1995). This "full" model is briefly summarized here since it will be used to investigate abnormal healing. It is based upon the following fundamental processes operating within the proliferative phase of healing (see also Olsen et al., 1995 and references therein for the biological basis of the model):

Fibroblasts. During wound healing, fibroblasts are actively motile, exhibiting both random and directed migration. These fluxes are modelled as 
unbiased Brownian motion for the former and as a chemotactic response to gradients in the growth-factor profiles for the latter. Fibroblasts are also passively convected by the strained tissue in which they reside.

New cells are generated by mitosis, modelled by the logistic growth function, with the mitotic rate substantially enhanced by the growth factors (which are cell cycle competence and progression factors). "Natural" cell senescence is described by simple linear decay. Finally, there are transformations between the two fibroblastic cell phenotypes. Within a dermal wound, a proportion of the fibroblasts undergo a reversible phenotypic change into contractile cells known as myofibroblasts. We model this transition by reversible kinetics, mediated by the growth-factor profile.

In addition, fibroblasts metabolize and secrete active growth factors, synthesize and degrade fibrillar extracellular matrix components (mediated by growth factors) and generate local traction stress within the tissue.

Myofibroblasts. These cells are not actively motile, but like fibroblasts, undergo passive convection by the moving tissue.

Cell proliferation and death are modelled as for the fibroblasts and the phenotypic transformations are the same, but with reversed signs. Note that the linear growth rate of myofibroblasts is lower than that for fibroblasts (Vande Berg et al., 1989) and that the death rate is increased due to "programmed" cell death, or "apoptosis" (Gabbiani, 1992).

Myofibroblasts also metabolize and secrete growth factors and synthesize and degrade extracellular matrix, possibly at a different rate to fibroblasts. Their roles in mediating fibroblast-generated traction forces are assumed to be transmission and amplification of these stresses within the extracellular matrix.

Growth factors. Many different families of inflammatory growth factors are known to regulate fibroplasia, but we consider a single, generic chemical (for simplicity) whose properties simulate the combined roles of in vivo promoters of healing such as platelet-derived growth factor and transforming growth factor $\beta$.

The chemical may diffuse through the tissue (again, modelled by Brownian motion) and is carried by passive convection. It is produced and metabolized by both cell types and undergoes tissue removal/inactivation, modelled as simple linear decay.

Extracellular matrix. Again, a wide variety of extracellular matrix components are involved in dermal wound healing, but we include only (fibrillar) type I collagen because of its central roles in fibroplasia and contraction. 
The only flux of the fibrillar collagen network is due to passive convection. It is synthesized (with growth-factor mediation and inhibition by high collagen concentrations) and degraded via specific enzymes by both cell types. The extracellular matrix also provides the support for cell-derived traction stresses and intrinsic tissue stresses.

2.2. Governing equations, boundary and initial conditions. With some further detail on the mathematical representations of these processes (Olsen et al., 1995) the non-dimensional model equations are derived from conservation laws for the fibroblast density $n(\mathbf{x}, t)$, myofibroblast density $m(\mathbf{x}, t)$, chemical concentration $c(\mathbf{x}, t)$ and collagen concentration $\rho(\mathbf{x}, t)$, where $\mathbf{x}$ is the spatial position vector and $t$ denotes time.

For simplicity, we consider a linear (or "slash") wound. Assuming the model variables to be independent of wound depth, this requires only a single spatial coordinate, $x$ say, in the plane of the skin. In this case the model equations are

$$
\begin{aligned}
& \frac{\partial n}{\partial t}=\overbrace{\frac{\partial}{\partial x}\left[D_{n} \frac{\partial n}{\partial x}-\frac{\alpha}{(\beta+c)^{2}} n \frac{\partial c}{\partial x}-n \frac{\partial u}{\partial t}\right]}^{\text {cell movement }}+\overbrace{\sigma\left(1+\frac{A c}{B+c}\right) n(1-\gamma n)}^{\text {mitosis }} \\
& -\underbrace{\frac{\kappa_{1} c}{C+c} n+\kappa_{2} m}_{\begin{array}{c}
\text { phenotypic } \\
\text { conversion }
\end{array}}-\underbrace{\delta n}_{\text {death }} \text {, } \\
& \frac{\partial m}{\partial t}=\overbrace{\frac{\partial}{\partial x}\left[-m \frac{\partial u}{\partial t}\right]}^{\text {convection }}+\overbrace{\epsilon_{\sigma} \sigma\left(1+\frac{A c}{B+c}\right) m(1-\gamma m)}^{\text {mitosis }} \\
& +\underbrace{\frac{\kappa_{1} c}{C+c} n-\kappa_{2} m}_{\substack{\text { phenotypic } \\
\text { conversion }}}-\underbrace{\lambda \delta m}_{\text {death }} \\
& \frac{\partial c}{\partial t}=\overbrace{\frac{\partial}{\partial x}\left[D_{c} \frac{\partial c}{\partial x}-c \frac{\partial u}{\partial t}\right]}^{\text {diffusion and convection }}+\overbrace{\frac{\kappa_{c}(n+\zeta m) c}{\gamma_{c}+c}}^{\text {production by cells }}-\overbrace{\delta_{c} c}^{\text {decay }} \\
& \frac{\partial \rho}{\partial t}=\overbrace{\frac{\partial}{\partial x}\left[-\rho \frac{\partial u}{\partial t}\right]}^{\text {convection }}+\overbrace{\left[\omega\left(1+\frac{P c}{Q+c}\right) \frac{1}{\phi^{2}+\rho^{2}}-\delta_{\rho} \rho\right](n+\eta m)}^{\text {synthesis and degradation by cells }} .
\end{aligned}
$$


Here, all parameters are positive, and typical values are given in the legend to Fig. 1. Note the fibroblast/myofibroblast phenotypic conversions, given by reversible dynamics in equations (1) and (2), with chemical mediation of the myofibroblastic differentiation. Note also that the chemical production term in equation (3) has a maximal rate related to the parameter $\kappa_{c}$.

All of the above processes which involve chemical mediation are modelled by the monotonically increasing and saturating Michaelis-Menten dynamics. This functional form is intuitively sensible, but is also derived from a theoretical analysis of cell-surface receptor binding dynamics in fibroblastic cell-growth-factor interactions (Olsen et al., 1995; Raines et al.; Sherratt et al., 1993).

In reality, unbiased cell movement may also depend upon the local growth-factor concentration (a phenomenon termed "chemokinesis"): this may be modelled by setting $D_{n}=D_{n}(c)$, but does not yield qualitative results different from those presented in this paper. Previous models of epidermal wound closure have shown chemokinesis to be an unimportant effect (see Dale et al., 1994, for example), and so we neglect this detail here.

A conservation law for the tissue momentum, together with simplifying assumptions that the tissue is a viscoelastic deformable continuum with a linear constitutive relation and negligible inertia, yields the fifth model equation governing tissue displacement, $\mathbf{u}(\mathbf{x}, t)$. In the one-dimensional linear geometry, this is

$$
\overbrace{\mu \frac{\partial^{3} u}{\partial x^{2} \partial t}}^{\text {viscous stress }}+\overbrace{\frac{\partial^{2} u}{\partial x^{2}}}^{\text {elastic stress }}+\frac{\overbrace{\nu \frac{\partial}{\partial x}\left[\frac{(1+\xi m) n \rho}{\psi^{2}+\rho^{2}}\right]}^{\text {active cell traction stress }}}{\text { resistance }} \text {. }
$$

The first two terms on the left-hand side of equation (5) arise from the viscous and elastic contributions to the intrinsic tissue stress tensor. The third term models the active cell traction stress, which is proportional to cell density and inversely proportional to the collagen concentration (for sufficiently large $\rho$ ), in agreement with theoretical and experimental considerations (Bell et al., 1979; Murray et al., 1988). Fibroblasts are assumed to generate the traction stresses, whereas myofibroblasts transmit and amplify these stresses within the extracellular matrix. The term on the right-hand side of (5) represents the (linear) restoring force which resists dermal tissue motion, which is assumed to be proportional to the displacement $u$ per unit collagen concentration. The parameter $s$ thus accounts for 
dermal tethering - it assumes lower values for loose-skinned mammals (such as rats and rabbits) and higher values for tight-skinned mammals (such as pigs and humans).

The wound centre is at $x=0$ with the initial wound margin given by $x=1$. By symmetry, these equations may be solved over the semi-infinite domain $0 \leq x, t<\infty$ with symmetric boundary conditions at $x=0$ and $\mathbf{q}=(n, m, c, \rho, u) \rightarrow \mathbf{q}_{0}=\left(n_{0}, 0,0, \rho_{0}, 0\right)$ as $x \rightarrow \infty$. Throughout this paper, $\mathbf{q}$ denotes the vector of model variables.

The vector $\mathbf{q}_{0}$ is a uniform steady state representative of normal dermis in which the myofibroblast and gencric growth-factor levels arc zero. By the scalings used in the non-dimensionalization process, $n_{0}$ and $\rho_{0}$ are set to unity (Olsen et al., 1995). The initial wound state, $\mathbf{q}_{\text {init }}(x)=$ $\left(n_{\text {init }}, 0, c_{\text {init }}, \rho_{\text {init }}, 0\right)$ is a large perturbation of the dermal state $\mathbf{q}_{0}$. Specifically, the initial conditions corresponding to a dermal excisional wound are as given in the following list:

Fibroblasts: None inside the wound and unwounded dermal levels outside, so $n_{\text {init }}(x)=H(x-1)$, where $H(\cdot)$ is the Heaviside step function.

Chemical: Active growth-factor profile centred around the wound site due to the early inflammatory response following injury. Specifically, we use the function $c_{\text {init }}(x)=c_{i} \exp \left(-x^{2}\right)$, which has an appropriate qualitative form.

Collagen: Low-level matrix deposition inside the wound during the early inflammatory phase and unwounded dermal concentration outside, giving $\rho_{\text {init }}(x)=\rho_{i}+\left(1-\rho_{i}\right) H(x-1)$.

The non-dimensional model parameter value estimates, sensitivity analyses and biological interpretations are detailed in Olsen et al. (1995), but some key parameters are highlighted as required in this paper.

2.3. Pathological steady states. Under normal conditions $\mathbf{q}_{0}$ is a locally stable steady state and the initial wound state evolves back to $\mathbf{q}_{0}$, thus simulating fibroplasia and contraction over a time scale of about 30 days, which is characteristic of the proliferative phase of healing. An example simulation of normal healing is illustrated in Fig. 1 (only the fibroblast density and tissue displacement is shown).

More generally, steady states $\mathbf{q}^{*}$ are the time-independent solutions of equations (1)-(5). The passive convection terms are zero at any steady state, so that the values of $\rho$ and $u$ are governed by the values of $n, m$ and 

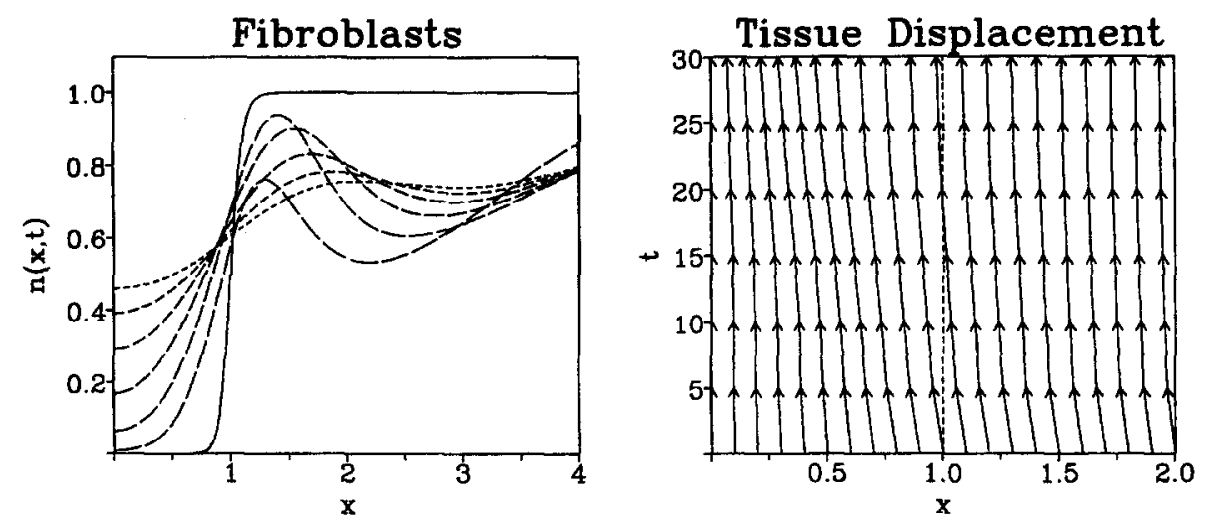

Figure 1. Numerical solution (using the methods of lines and Gear's method) of the "full" model given by equations (1)-(5) with boundary and initial conditions as in section 2.2. This simulation corresponds to normal healing. Profiles of fibroblast cell density and tissue displacement only are plotted against distance $x$ from the wound centre at six successive time intervals of 5 days. For the fibroblasts, these are indicated by increasingly broken curves. Tissue displacement is represented as the movement of (initially evenly spaced) tattoo marks in and around the wound site with time, as indicated by the arrows. The initial wound margin is $x=1$ (given by the dashed line in the tissue displacement picture) and a finite domain approximation $x_{\infty}=20$ is used for these simulations, with zero flux imposed at $x_{\infty}$ rather than dermal steady state values. Only $x \in[0,4]$ is shown, for clarity. These simulations show that during normal healing, fibroblasts infiltrate the wound space and tissue contraction occurs in and around the wound site. Parameter values are $D_{n}=0.02, \alpha=0.1, \beta=0.2$, $\sigma=0.02, A=44.5, B=1, \gamma=0.01, \kappa_{1}=10, C=1, \kappa_{2}=1, \delta=0.0198, \epsilon_{g}=0.5$, $\lambda=10, D_{c}=0.2, \kappa_{c}=0.4, \zeta=1, \gamma_{c}=1, \delta_{c}=0.5, \omega=0.008, P=10, Q=0.1$, $\phi=3, \delta_{p}=0.0008, \eta=2, \mu=20, \nu=0.03, \xi=5, \psi=0.005, s=1, c_{i}=1$ and $\rho_{i}=0.01$. Note that $n_{0}=1$ and $\kappa_{c}^{(2)}=0.5$.

$c$, whose steady state equations are decoupled from those of $\rho$ and $u$. Restricting attention to spatially uniform steady states yields the equations

$$
\begin{array}{r}
\sigma\left(1+\frac{A c}{B+c}\right) n(1-\gamma n)-\frac{\kappa_{1} c n}{C+c}+\kappa_{2} m-\delta n=0, \\
\epsilon_{\sigma} \sigma\left(1+\frac{A c}{B+c}\right) m(1-\gamma m)+\frac{\kappa_{1} c n}{C+c}-\kappa_{2} m-\lambda \delta m=0, \\
\frac{\kappa_{c}(n+\zeta m) c}{\gamma_{c}+c}-\delta_{c} c=0
\end{array}
$$

for $n, m$ and $c$.

In addition to the normal dermal steady state $\mathbf{q}_{0}=\left(n_{0}, 0,0, \rho_{0}, 0\right)$, equations (6)-(8) also permit solutions for which $n>n_{0}, m>0$ and $c>0$, which is highly indicative of a pathologically excessive healing response. This is 

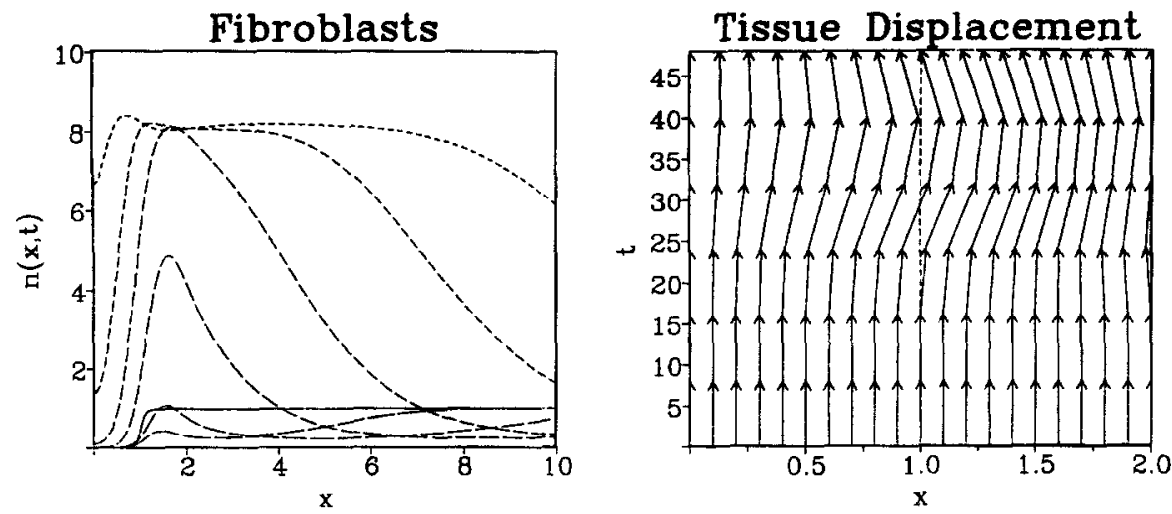

Figure 2. Numerical solution of the "full" model as in Fig. 1, but plotted at successive time intervals of 8 days. This simulation corresponds to a pathological response; fibroblast density becomes elevated above the normal dermal level and considerable tissue forces are generated (note that in this simulation, the cell traction parameter $\nu$ was decreased in order to yield mathematically valid solutions - see below and Fig. 1 legend). The apparent outward tissue strain is due to the "tugging" effect at the leading edge of the advancing pathology, and is followed by contraction of the affected tissue behind the front. In addition (not shown), myofibroblast, chemical and collagen levels also become substantially and permanently increased above their normal values, suggestive of the type of fibro-proliferative disorder introduced in section 1. A wider spatial domain, $x \in[0,10]$, is shown here. Parameter values as in Fig. 1 except $\kappa_{c}=1$ and $\nu=0.002$.

evident from numerical solutions of (6)-(8) for a wide range of biologically valid parameter values, and some "pathological" steady states, $\mathbf{q}_{p}$ say, can be shown to be locally stable to small perturbations. Figure 2 shows a typical pathological simulation.

Significantly, equations (6)-(8) imply that if $c>0$ and $n>0$, then $m \neq 0$, so that any pathological state with a persistent inflammatory component (indicated by $c>0$ ) exhibits a permanent myofibroblastic profile, which is a paradigm of an important class of fibro-proliferative pathologies (Ehrlich et al., 1994; McCann et al., 1993; Murray, 1993; Rudolph and Vande Berg, 1991; Schürch et al., 1990; Skalli and Gabbiani, 1988).

3. Analysis of a Caricature Model. The model has been shown to exhibit steady solutions reminiscent of healing pathologies. Biologically, it is crucial to determine the key parameters which regulate the transformation between normal and abnormal healing. Equations (6)-(8) are not explicitly solvable, however, so to gain further insight into the steady states of (1)-(5) and the dynamics of pathological and normal healing responses we propose a caricature model which mimics the essential features of the full system. 
3.1. Derivation of the caricature model. Explicitly, we consider a composite cell density $n(x, t)$ representing both fibroblasts and myofibroblasts, and a generic chemical concentration $c(x, t)$ with similar properties to those described in section 2.1:

Cells. Since the tissue biomechanics have been omitted, passive convection is neglected and the cell flux is due to random migration and chemotaxis. The cell proliferation and loss terms are the same as in the full model, but there are no phenotypic conversions because of the assumption of fibroblastic homogeneity.

Chemical. Diffusion is the only contribution to the chemical flux, as there is no convection. The cells produce the chemical, with the same metabolic dependence on chemical concentration as in the full model, and there is also tissue removal/inactivation.

As in the full model, chemical mediation of cell chemotaxis, proliferation and production of the chemical obeys the Michaelis-Menten uptake form based on the dynamics of growth factors and their cell-surface receptors.

The equations. The above processes capture the fundamental fibroblastic cellular functions and their coupling to the growth-factor dynamics of the full modcl as in equations (1)-(3), giving the caricature system

$$
\begin{aligned}
& \frac{\partial n}{\partial t}=\overbrace{\frac{\partial}{\partial x}\left[D_{n} \frac{\partial n}{\partial x}-\frac{\alpha}{(\beta+c)^{2}} n \frac{\partial c}{\partial x}\right]}^{\text {cell movement }} \\
& \overbrace{\sigma\left(1+\frac{A c}{B+c}\right) n(1-\gamma n)}^{\text {mitosis }}-\overbrace{\delta n}^{\text {death }} \equiv f(n, c) \text {, } \\
& \frac{\partial c}{\partial t}=\overbrace{D_{c} \frac{\partial^{2} c}{\partial x^{2}}}^{\text {diffusion }}+\frac{\overbrace{\kappa_{c} n c}^{\text {production }}}{\gamma_{c}+c}-\overbrace{\delta_{c} c}^{\text {decay }} \equiv g(n, c) \text {. }
\end{aligned}
$$

As in section 2.2, the boundary conditions are zero species gradients at $x=0$ by symmetry and $\mathbf{q}=(n, c) \rightarrow \mathbf{q}_{0}=\left(n_{0}, 0\right)$ as $x \rightarrow \infty$. Again, $\mathbf{q}_{0}$ is interpreted as the normal dermal steady state and $n_{0}=[1-\delta / \sigma] / \gamma$ is set to unity. The initial wound state is given by $\mathbf{q}_{\text {init }}(x)=\left(H(x-1), c_{\text {init }}(x)\right)$, 
obtained by analogous arguments for the initial cellular and chemical profiles as in the full model.

3.2. Existence and stability of steady states. Uniform steady states $\mathbf{q}^{*}$ of (9) and (10) are the solutions of $f(n, c)=0=g(n, c)$. These, together with the parameter inequalities which determine their existence (equivalent to non-negative values for the species variables) and stability, can be deduced analytically:

Trivial: $q_{\mathrm{t}}=(0,0)$. With no cells or growth factors, this steady state is expected intuitively and may be representative of a chronic non-healing wound scenario; however, it is of little relevance to excessive healing pathologies, the focus of this paper. The steady state exists for all parameter values and is locally stable if and only if $\sigma<\delta$.

Dermal: $\boldsymbol{q}_{0}=\left(n_{0}, 0\right)$. Here there are fibroblasts, but no active growth factors. This dermal, or unwounded, steady state exists if and only if $\delta<\sigma$, so this constraint must be satisfied by realistic parameter values. $\mathbf{q}_{0}$ is locally stable if and only if $\delta<\sigma$ and $\kappa_{c} n_{0}<\gamma_{c} \delta_{c}$.

Pathological: $\boldsymbol{q}_{p}=\left(n_{p}, c_{p}\right)$. Steady states with $\mathbf{q}_{p}>\mathbf{0}$ are not quite as simple: $c_{p}$ is given by real, positive roots of $p(c) \equiv a_{2} c^{2}+a_{1} c+a_{0} \equiv 0$, where $a_{2}=(1+A) \delta_{c}, a_{1}=(1+A)\left(\gamma_{c} \delta_{c}-\kappa_{c} n_{0}\right)+A \kappa_{c}\left(n_{0}-\gamma^{-1}\right)+B \delta_{c}$ and $a_{0}=B\left(\gamma_{c} \delta_{c}-\kappa_{c} n_{0}\right)$. Also, $g=0$ implies $n_{p}=\delta_{c}\left(\gamma_{c}+c_{p}\right) / \kappa_{c}$. The necessary and sufficient condition for one such steady state is $a_{0}<0$ and those for two pathological steady states $\left(\mathbf{q}_{ \pm}\right.$, say, where $\left.\mathbf{q}_{+}>\mathbf{q}_{-}\right)$are $a_{0}>0, a_{1}<0$ and $a_{1}^{2}-4 a_{0} a_{2}>0$. Otherwise, there are no such solutions.

Linear stability analysis together with the signs of $f$ and $g$ in the $(n, c) \geq(0,0)$ quadrant of phase space show that when only one pathological steady state exists, it is a stable node and $\mathbf{q}_{0}$ is a saddle point. If both $\mathbf{q}_{ \pm}$exist, however, then $\mathbf{q}_{-}$is a saddle point and both $\mathbf{q}_{0}$ and $\mathbf{q}_{+}$are stable nodes. The regions of attractions of $\mathbf{q}_{0}$ and $\mathbf{q}_{+}$are delineated by the separatrix, $n=S(c)$ say, which is also the stable manifold through $\mathbf{q}_{-} . S(c)$ is confined to the positive quadrant and is a monotonic decreasing function. These results are summarised qualitatively in Fig. 3.

3.3. Key parameters and bifurcations. The values of parameters in the inequality $\kappa_{c} n_{0}<\gamma_{c} \delta_{c}$ (except $n_{0}$, which is set to unity) are crucial in determining the outcome of the healing response. Note that the intercept of the $g=0$ null cline on the $n$-axis is $\gamma_{c} \delta_{c} / \kappa_{c} n_{0}$ (Fig. 3). As $\kappa_{c}$ is 


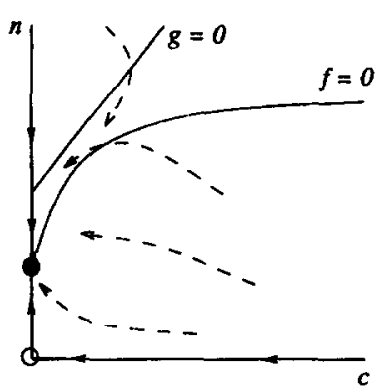

(a)

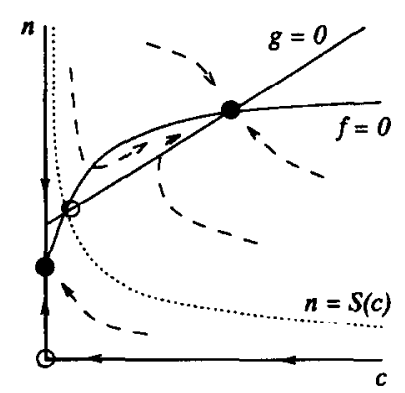

(b)

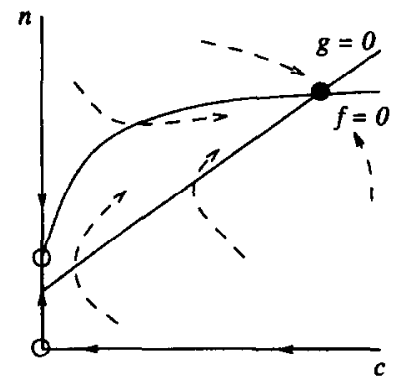

(c)

Figure 3. Qualitative phase space diagrams for the caricature model with kinetics $f$ and $g$ as in (9) and (10), respectively (section 3.1). The positive quadrant null clines $f=0$ and $g=0$ and the biologically realistic steady states $\mathbf{q}^{*}$ arc shown, with fillcd circles represcnting (locally) stable steady states and open circles representing unstable steady states. In (a), the dermal steady state $\mathbf{q}_{0}$ is globally stable. In (b), $\mathbf{q}_{0}$ and the pathological steady state $\mathbf{q}_{+}$are locally stable whereas the pathological steady state $\mathbf{q}_{-}$is unstable - the attracting regions of $\mathbf{q}_{0}$ and $\mathbf{q}_{+}$are separated by the stable manifold through the saddle point at $\mathbf{q}_{-}$. In (c), $\mathbf{q}_{+}$is globally stable.

sequentially increased (or as $\gamma_{c}$ or $\delta_{c}$ is decreased) whilst fixing the other parameter values, the dynamics exhibit two important bifurcations. This is qualitatively evident from Fig. 3 , but also from the steady state equations $f(n, c)=0=g(n, c)$ and their linear stability analyses.

We focus on the parameter $\kappa_{c}$ for the remainder of this paper. This parameter represents the maximal rate of active growth-factor production by fibroblastic cells, since this is a potentially manipulable biological parameter. Much of the following analysis, however, could be pursued for either $\delta_{c}$, the rate of active growth-factor removal from the tissue, or $\gamma_{c}$, the active growth-factor concentration which elicits half-maximal binding of cellsurface receptors to their growth-factor ligands.

(i) Saddle-node bifurcation at $\kappa_{c}=\kappa_{c}^{(1)} . \quad \kappa_{c}^{(1)}$ is calculated from the equation $a_{1}^{2}-4 a_{0} a_{2}=0$ under the constraints $a_{0}>0$ and $a_{1}<0$ (section 3.2). As $\kappa_{c}$ increases through $\kappa_{c}^{(1)}, \mathbf{q}_{0}$ remains locally stable but loses its global stability as the pathological steady states $\mathbf{q}_{-}$and $\mathbf{q}_{+}$appear, which are unstable and locally stable, respectively (Fig. 3(a, b)).

(ii) Transcritical bifurcation at $\kappa_{\mathrm{c}}=\kappa_{\mathrm{c}}^{(2)} . \quad \kappa_{c}^{(2)}$ is easily calculated to be $\gamma_{c} \delta_{c} / n_{0}$. As $\kappa_{c}$ increases through $\kappa_{c}^{(2)}, \mathbf{q}_{0}$ loses stability and $\mathbf{q}_{+}$becomes globally stable (Fig. 3(b, c)).

The dynamics of $n$ and $c$ may be summarized in a bifurcation diagram, obtained by plotting the steady states $n^{*}$ and $c^{*}$ as functions of $\kappa_{c}$ as 

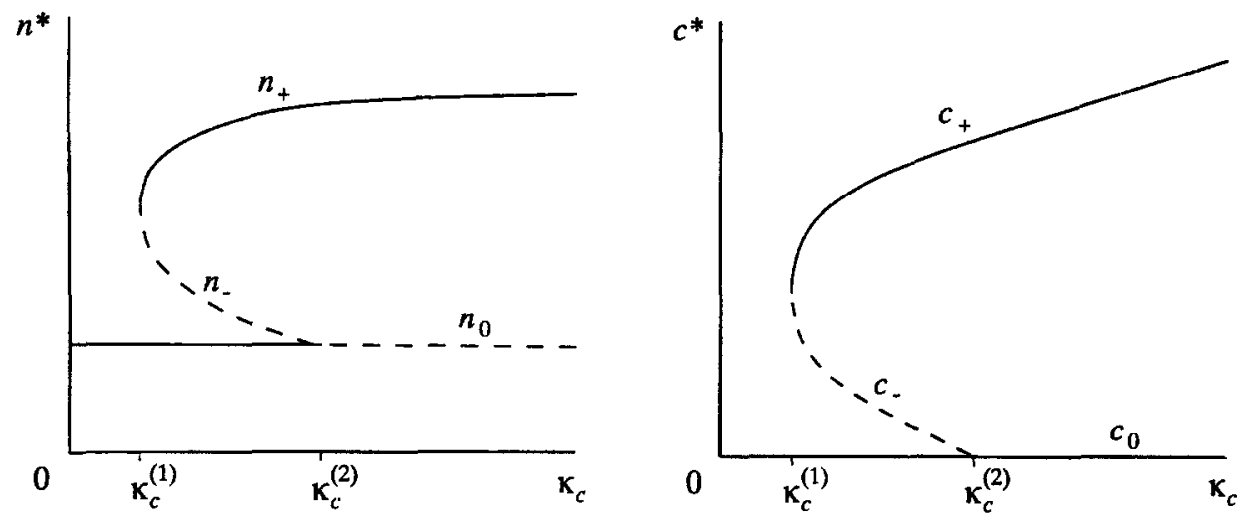

Figure 4. Qualitative bifurcation diagrams illustrating the variations of the steady states $\left(n^{*}, c^{*}\right)$ with the parameter $\kappa_{c}$, as deduced from the analysis of sections 3.2 and 3.3 (see also Fig. 3). Solid curves represent (locally) stable steady states and broken curves represent unstable steady states. The dermal steady state $\left(n_{0}, c_{0}\right)$ and the pathological steady states $\left(n_{ \pm}, c_{ \pm}\right)$are indicated.

shown in Fig. 4. The qualitative dynamics of the system can be subdivided into three intervals: $I_{\text {norm }}=\left(0, \kappa_{c}^{(1)}\right), I_{\text {bist }}=\left(\kappa_{c}^{(1)}, \kappa_{c}^{(2)}\right)$ and $I_{\text {path }}=\left(\kappa_{c}^{(2)}, \infty\right)$.

If $\kappa_{c} \in I_{\text {norm }}$, then the dynamics represent normal wound healing, for any initial conditions (see also Fig. 3(a)). If $\kappa_{c} \in I_{\text {path }}$, then a pathological transition will occur - again, given any initial data (see also Fig. 3(c)). The bistable regime, $\kappa_{c} \in I_{\text {bist }}$, however, exhibits richer behaviour (see also Fig. 3(b)). Here, the initial conditions and other parameter values strongly influence the dynamics of the system (9) and (10). The dependence on initial profiles is crucial and means that some wound conditions may trigger a pathological response while others do not.

The bistability in this system (for $\kappa_{c} \in I_{\text {bist }}$, see Fig. 4) has important consequences. Suppose that $\kappa_{c} \in I_{\text {bist }}$ and that normal healing dynamics are in progress. If a forced perturbation to the system causes $(n, c)$ to move from the attracting region for $q_{0}$ to that for $q_{+}$, then there will be a pathological outcome unless another parameter, such as $\kappa_{c}$, is also changed. Similarly, if a forced perturbation increases $\kappa_{c}$ above $\kappa_{c}^{(2)}$, then $\kappa_{c}$ must be lowered below $\kappa_{c}^{(1)}$ to ensure normal healing. Biologically, these predictions suggest various therapeutic strategies, as discussed later.

3.4. Spatiotemporal development of pathological states. The spatial nature of normal and pathological healing dynamics is of great interest. First, normal healing is simulated by solving equations (9) and (10) with boundary and initial conditions as in section 3.2. Typical results are shown in Fig. 5, in which $\kappa_{c} \in I_{\text {bist }}$ and the initial data lie entirely within the attracting 

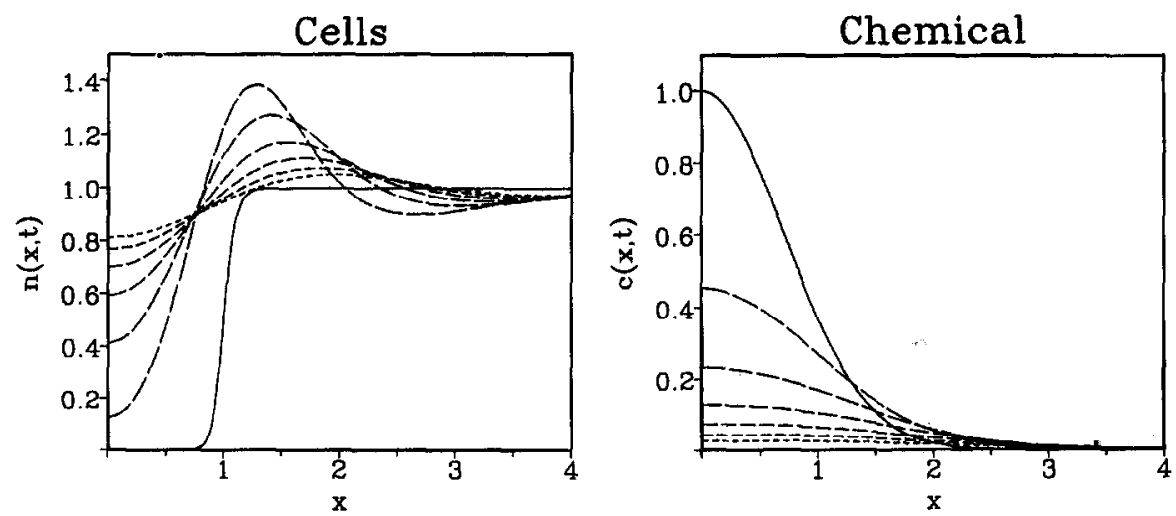

Figure 5. Numerical solution of the caricature model given by equations (9) and (10) and boundary and initial conditions as in section 3.2 , corresponding to normal healing. Profiles of cell density and chemical concentration are plotted against distance $x$ from the wound centre at six successive time intervals (given by increasing broken curves) of 5 days (cells) and 1 day (chemical). See also Fig. 1. Parameter values are $D_{n}=0.02, \alpha=0.05, \beta=0.2, \sigma=0.02, A=44.5, B=1$, $\gamma=0.01, \delta=0.0198, D_{c}=0.2, \kappa_{c}=0.1, \gamma_{c}=1, \delta_{c}=0.5$, and $c_{i}=1$. Again, $n_{0}=1$ and $\kappa_{c}^{(2)}=0.5$.

region for $\mathbf{q}_{0}$. Normal healing is characterised by chemical-driven cellular proliferation into the wound space $(0 \leq x \leq 1)$ accompanied by a relatively rapid chemical decay (Fig. 5).

If $\kappa_{c} \in I_{\text {path }}$, however, the numerical simulations show markedly different behaviour, as demonstrated in Fig. 6, which is suggestive of a severe
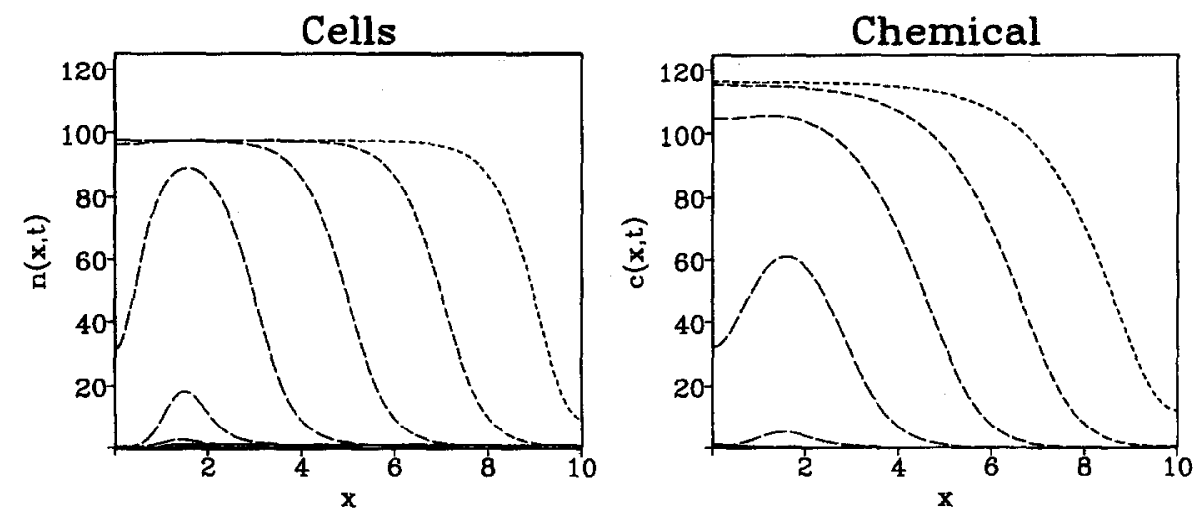

Figure 6. Numerical solution of the caricature model as in Fig. 5, but with successive time intervals of 5 days for both the cell and chemical profiles, and $\kappa_{c}=0.6>\kappa_{c}^{(2)}$. This simulation corresponds to a severe healing pathology, characterised by grossly excessive cellular and chemical levels which propagate from near the original wound margin. See also Fig. 2. 
fibro-proliferative pathology (section 1). Wave-like solutions appear to evolve from a point near the initial wound boundary, $x=1$, resulting in highly elevated cell and chemical levels which not only invade the wound space, but also spread into previously unaffected tissue over a time scale of days.

Pathological responses arising from $\kappa_{c} \in I_{\text {bist }}$ (as discussed above) exhibit similar spatiotemporal behaviour to that illustrated in Fig. 6 (not shown).

From the form of equations (9) and (10) and the numerical simulations of the pathological response, it appears that travelling wave solutions (that is, with constant wave velocity and wave profiles) develop from the initial conditions. Such solutions have the form $n(x, t)=N(z)$ and $c(x, t)=C(z)$, where $z=x-a t$ and $a$ is the wave velocity. Substituting into (9) and (10) gives a system of coupled ordinary differential equations.

The supposition that travelling wave solutions effect a transition from the dermal steady state $\mathbf{Q}_{0}$ to the pathological steady state $\mathbf{Q}_{+}$is true if and only if there exists a phase trajectory, $\mathscr{T}$ say, between $\mathbf{Q}_{0}$ and $\mathbf{Q}_{+}$in $\mathbf{Q}=(N, C, U, V)$ space, where $U=\mathrm{d} N / \mathrm{d} z$ and $V=\mathrm{d} C / \mathrm{d} z$ (see Murray, 1989, for several examples of travclling wave analyses). Further, Fig. 6 shows that $\mathbf{Q} \rightarrow \mathbf{Q}_{0}$ as $z \rightarrow \infty$ and that $\mathbf{Q} \rightarrow \mathbf{Q}_{+}$as $z \rightarrow-\infty$. Thus, necessary conditions for $\mathscr{T}$ to exist are a stable local manifold at $\mathbf{Q}_{0}$ and an unstable local manifold at $\mathbf{Q}_{+}$.

Local stability analyses of the system in $\mathbf{Q}$-space at $\mathbf{Q}_{0}$ and $\mathbf{Q}_{+}$reveal that for $\kappa_{c} \in I_{\text {path }}$, these conditions are satisfied. The further requirement that $\mathscr{T}$ must lie entirely in the subspace $(N, C) \geq(0,0)$ imposes a lower bound on $a$ in terms of the model parameters:

$$
a \geq a_{\min }=2 \sqrt{D_{c}\left(\frac{\kappa_{c} n_{0}}{\gamma_{c}}-\delta_{c}\right)} .
$$

For $\kappa_{c} \in I_{\text {bist }}$, the above conditions are again satisfied, but no minimum wave velocity is stipulated in this case.

In summary, travelling wave solutions of (9) and (10) may drive the system to the stable pathological steady state if and only if $\kappa_{c}>\kappa_{c}^{(1)}$, and that this state invades neighbouring tissue with a minimum wave velocity of $a_{\min }$ if $\kappa_{c}>\kappa_{c}^{(2)}$. It can be shown that if either cell chemotaxis or random migration is neglected, by setting $\alpha$ or $D_{n}$ to zero, respectively, in (9), then the basic methods and results of the travelling wave analysis as outlined above are unaffected.

Whether active extracellular growth factors are freely diffusible through tissue is unclear - the availability of active growth factors in vivo may be mediated by ligand binding to fixed sites within the fibrillar extracellular 
matrix scaffold (Clark, 1991). This scenario is modelled by setting $D_{c}$ to zero in equation (10). A similar analysis to that above predicts that travelling wave solutions may exist for any $\kappa_{c}>\kappa_{c}^{(1)}$, but no minimum wave velocity is imposed. In this case, numerical simulations reveal that pathological waves do form (as for the case $D_{c}>0$ ) and that this depends on the initial conditions when $\kappa_{c} \in I_{\text {bist }}$.

3.5. Cessation and regression. Having investigated the initiation and progression of pathological responses in relation to normal healing dynamics, two important clinical problems remain. First, how do invasive pathological dynamics become spatially limited? Fibro-proliferative pathologies rarely proceed far beyond the original wound boundaries, except in excessive dermal scars such as keloids (Asmussen and Söllner, 1993; Murray, 1993), whereas the model predicts that a pathological wave will advance (with constant speed) throughout the skin. Second, what mechanisms might cause a fibro-proliferative disorder to partially or fully regress? The clinical occurrence, extent and permanence of regression is unpredictable and unexplained (McCann et al., 1993; Murray and Pinnell, 1992). These issues are addressed below.

(i) Cessation. The phenomenon of a wave-like progression of a pathological state (Fig. 6) followed by a cessation of the wave resulting in a stable, spatially bounded disease would correspond to a stable spatially inhomogeneous steady state solution of equations (9) and (10). Such a solution is only possible if some model parameters are spatially inhomogeneous such that $\mathbf{q}_{+}$is locally stable near $x=0$ and unstable for large $x$.

Focusing again on $\kappa_{c}$, this implies that $\kappa_{c} \in I_{\text {norm }}$ for large $x$, and Fig. 7 illustrates the solution of the caricature model with such a spatially varying $\kappa_{c}$.

Figure 7 confirms that the pathological travelling wave is halted approximately at the point $x=x_{b}$ where $\kappa_{c}$ falls below $\kappa_{c}^{(1)}$, although there is substantial "overshoot," most notably in cellular levels, due to the chemical diffusive flux which effectively modulates the sharp transition of $\kappa_{c}$ through its bifurcation value. This is confirmed by setting $D_{c}$ to zero and repeating the simulations. In this case, the wave stops abruptly at $x_{b}$ with no overshoot (not shown).

Note that if $\kappa_{c}(x) \in I_{\text {path }}$ for $0 \leq x \leq x_{b}$ but $\kappa_{c}(x) \in I_{\text {bist }}$ for $x>x_{b}$, then the pathological wave velocity and profiles will become modulated at $x_{b}$ but cessation does not occur because $\mathbf{q}_{+}$remains locally stable.

The implications of the above results are discussed in section 5 . 

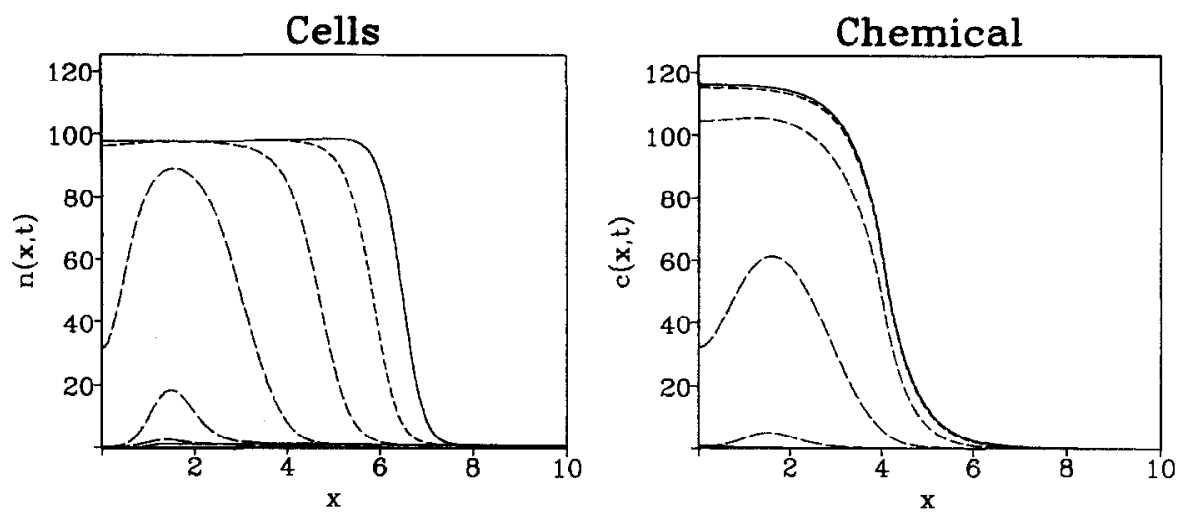

Figure 7. Numerical solution of the caricature model as in Fig. 6, but with $\kappa_{c}(x)=0.6$ if $0 \leq x \leq 4$ and $\kappa_{c}(x)=0$ if $x>4$. The solid curves are the steady state profiles (calculated at 150 days). Note that the spatial extent of the pathology extends beyond the value $x_{b}=4$ (see text).

(ii) Regression. A related problem concerns the generally variable regression of some fibro-proliferative disorders. In the context of the caricature model, such behaviour is represented by pathological dynamics toward $\mathbf{q}_{+}$from the initial conditions, followed in time by wave cessation and a gradual return toward $\mathbf{q}_{0}$ throughout the domain.

Whereas cessation is an inherently spatial problem, regression is essentially a temporal anti-pathogenic phenomenon. Such a mechanism could involve time-dependent model parameter values-a reduction in $\kappa_{c}$, for example. By analogy with the above arguments for cessation, regression is possible if $\kappa_{c}(t) \in I_{\text {norm }}$ for all $t>t_{r}$, where $t_{r}$ is a characteristic regression time of the disease. This would imply that $\mathbf{q}_{+}$loses stability to $\mathbf{q}_{0}$ at time $t_{r}$, initiating a transition back toward the normal dermal state. Results for a simple switch $\kappa_{c}(t)=1.2 \kappa_{c}^{(2)}$ for $0 \leq t \leq t_{r}$ and $\kappa_{c}(t)=0$ for $t>t_{r}$ are shown in Fig. 8.

After time $t_{r}$, the regression in the chemical concentration is rapid, within a few days, whereas cell levels diminish much more gradually, over a time scale of years toward their dermal levels (Fig. 8), as is typical of keloid and hypertrophic scar regression (Murray and Pinnell, 1992). Note that the pathological response response progresses some distance beyond the point at which the temporal switch occurs and that the regression dynamics are monotonic rather than wave-like: these features are particularly evident from the cell profiles in Fig. 8. Again, overshoot does not occur for the case $D_{c}=0$ (not shown), and reducing $\kappa_{c}$ into the interval $I_{\text {bist }}$ only serves to modulate the advancing pathology rather than inducing regression. 

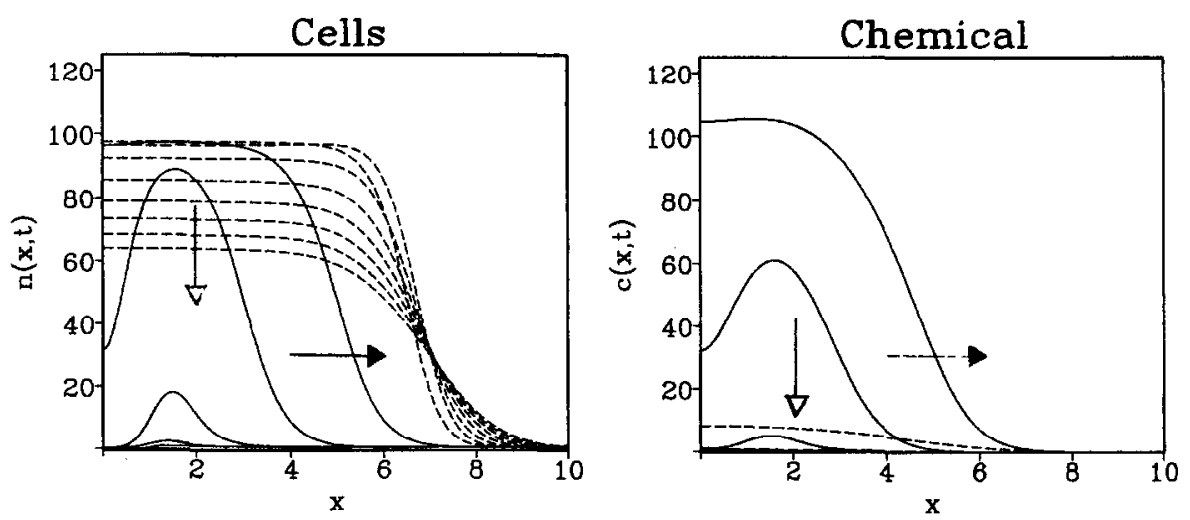

Figure 8. Numerical solution of the caricature model as in Fig. 6, but with 12 successive time intervals (each of 5 days) and $\kappa_{c}(t)=0.6$ if $0 \leq t \leq 20$ and $\kappa_{c}(t)=0$ if $t>20$. Solution profiles at times $0 \leq t \leq 20$ are indicated by solid curves and those for $t>20$ by broken curves for clarity. The pathological wave progression is indicated by solid arrows and the regression by empty arrows.

These non-autonomous forms for $\kappa_{c}(x, t)$ could, in reality, arise from factors not explicitly modelled in this work. For example, synergy between different types of growth factors (which are activated during wound healing) can cause one such chemical to up-regulate cellular production of another (Clark, 1991; Martin et al., 1992; Sprugel et al., 1987), perhaps by increasing cell-surface receptor expression or by promoting intracellular messenger signals involved in growth-factor-mediated biosynthesis.

4. Discussion. In this work, we have addressed some clinically important issues regarding fibro-proliferative disorders of dermal wound healing (outlined in section 1) by application of a previously proposed deterministic mathematical model for fibroplasia and wound contraction (Olsen et al., 1995) (section 2). A simpler, caricature model was developed to investigate a range of fibro-proliferative healing disorders characterized by excessive fibroblastic cell and inflammatory growth-factor profiles (sections 2.3 and 3.1). Qualitative and quantitative insight was obtained into the pathogenesis, progression, stability, cessation and regression of pathological states in relation to normal healing, highlighting the roles of key model processes and parameters (sections 3.2-3.5).

The dynamics $g(n, c)$ of the inflammatory growth-factor mediators are crucial to the behaviour of the model system given by equations (9) and (10) and, particularly, the balance between cellular production of the active chemical and chemical removal from the tissue is pivotal; the former is controlled by the parameter $\kappa_{c}$. Pathogenesis to a disease state is guaran- 
teed if $\kappa_{c}>\kappa_{c}^{(2)}$ and is possible, depending on the initial perturbation to the normal dermal state, if $\kappa_{c}>\kappa_{c}^{(1)}$. This is clear from the phase plane, linear stability and bifurcation analyses of sections 3.2 and 3.3 (see also Figs. 3 and 4). Transitions to pathological states are proposed to occur via travelling waves: one moving into the wound space (the "healing" wave) and another moving away from the wound (the "pathological" wave) into normal skin (Fig. 6, section 3.4). If $\kappa_{c}>\kappa_{c}^{(2)}$ and $D_{c}>0$, then the pathological wave has a minimum speed which we have calculated analytically.

The resulting pathology can become spatially bounded if $\kappa_{c}$ varies appropriately with distance, $x$, from the wound centre (section 3.5). Figure 7 illustrates this phenomenon, showing considerable "overshoot" of the pathological response. Similarly, gradual regression of the disease may occur if $\kappa_{c}$ varies appropriately with time $t$ (section 3.5), as demonstrated in Fig. 8. The difference between keloid and hypertrophic scarring-namely, that the latter condition generally remains confined within the original wound boundaries - is manifest in the spatial extent of the pathology, which in this model is determined by the form of $\kappa_{c}(x, t)$ and by other parameter values (the chemical diffusion coefficient $D_{c}$ influences the degree of the overshoot effect, for example).

The prescribed non-autonomous forms for $\kappa_{c}(x, t)$ (section 3.5) crudely account for processes involved in wound healing that have not been explicitly included in this model. Although $\kappa_{c}(x, t)$ may, in principle, be derived from an appropriate conservation-type equation, the additional complexity would question this approach within our "caricature" framework.

The effects of spatiotemporal changes in $\kappa_{c}$ may be understood by reference to the bifurcation diagram (Fig. 4). The bistability exhibited by the system for $\kappa_{c}^{(1)}<\kappa_{c}<\kappa_{c}^{(2)}$ is a crucial property of the model and has potentially important implications. For example, suppose that the dynamics lie on the stable branch $\mathbf{q}_{0}$. If $\kappa_{c}$ is increased beyond $\kappa_{c}^{(2)}$, then the dynamics move to the stable "pathological" branch $\mathbf{q}_{+}$. However, a therapeutic (or natural) reduction in $\kappa_{c}$ below $\kappa_{c}^{(2)}$ is insufficient: $\kappa_{c}$ must fall below $\kappa_{c}^{(1)}$ for the pathology to be successfully treated. Therapeutic and corrective strategies in combating fibro-proliferative disorders could benefit from new insights gained from these theoretical results.

We propose that adult human dermis of individuals has an effective $\kappa_{c}$ below $\kappa_{c}^{(2)}$ with the consequence that the dermal state is stable so that normal healing dynamics are initiated upon wounding (Fig. 5). This permits the scenario that $\kappa_{c}>\kappa_{c}^{(1)}$, however, in which case, certain wound conditions and trauma-induced local perturbations may trigger a pathological response (section 3.3). Such a hypothesis suggests mechanisms for the apparent differential incidences of fibro-proliferative disorders between 
racial groups and variations with age, anatomical location and other attributes (Asmussen and Söllner, 1993; Murray, 1993). The hypothesis may also explain the well known but inadequately understood observation that hypertrophic scarring occurs more frequently in full-thickness burns wounds than in excisional wounds (Asmussen and Söllner, 1993; Boykin and Molnar, 1992; Murray and Pinnell, 1992). The heightened inflammatory cell influx early in the healing of burn wounds may serve to increase the initial supply of active growth factors, thus triggering the excessive healing response. Pathogenic stimuli could also be related to cellular signals that either effectively enhance $\kappa_{c}$, perhaps by up-regulating ligand receptor expression in fibroblastic cells, or that deplete growth-factor inactivation sites in the extracellular milieu (Clark, 1991). There are also other possibilities, such as excessive supplies of activated growth factors or activation signals, or up-regulated binding protein levels which effectively prolong the efficacy of polypeptide growth factors in vivo (Bowen-Pope et al., 1984; Raines et al., 1990; Sprugel et al., 1987). These scenarios are all potentially testable, with the model predictions of section 3 borne in mind.

Onc important outstanding question concerns whether the caricature model (9) and (10) is an adequate representation of the full model system (1)-(5) with respect to the behaviour studied in section 3. Numerical simulations suggest that this is indeed the case. The full model can be shown to exhibit a steady state structure similar to that for the caricature model, as alluded to in sections 2.3-3.2. The advantage of the caricature model is that it can be studied analytically; in the full model we must rely on numerical solutions alone. The full model elicits the same qualitative phenomena of pathogenesis, wave-like progression, cessation and regression as the caricature model, but also yields insight into the extracellular matrix involvement in these diseases. Specifically, excessive collagen accumulation occurs in the pathological response-which is expected from equation (4) - and intense mechanical forces ("contractures") are generated, particularly at the leading front of the disease (not shown). These biological features are typical of fibro-proliferative disorders (Boykin and Molnar, 1992; Flint, 1990; Murray, 1993; Rudolph et al., 1992; Skalli and Gabbiani, 1988).

The fundamental conclusion of this work is that with an understanding of the spatiotemporal dynamics of fibro-proliferative disorders, we may suggest mechanisms by which these pathologies arise, develop, stabilize and regress, and propose strategies for new clinical approaches in managing these diseases - all of which are potentially testable in experiments.

LO expresses gratitude to the Engineering and Physical Sciences Research Council of Great Britain for a Research Studentship award to support this 
work. Part of this work was carried out while PKM was visiting the School of Mathematics and Statistics, University of Sydney, Australia, and the Department of Mathematics, Williams College, Massachusetts, U.S.A. This work was supported in part by a grant from the London Mathematical Society. We would like to thank Dr. Frank Arnold and Dr. George Cherry (Dermatology Unit, Churchill Hospital, Oxford, UK) and Rebecca Hollands (Department of Physics, Surrey University, UK) for helpful discussions.

\section{REFERENCES}

Asmussen, P. D. and B. Söllner. 1993. Wound Care. Principles of Wound Healing. Hamburg: Beiersdorf Medical Bibliothek.

Bell, E., B. Ivarsson and C. Merrill. 1979. Production of a tissue-like structure by contraction of collagen lattices by human fibroblasts of different proliferative potential in vitro. Proc. Natl. Acad. Sci. USA 76, 1274-1278.

Bowen-Pope, D. F., T. W. Malpass, D. M. Foster and R. Ross. 1984. Platelet-derived growth factor in vivo: levels, activity and rate of clearance. Blood 64, 458-469.

Boykin, J. V. and J. A. Molnar. 1992. Burn scar and skin equivalents. In Wound Healing: Biochemical and Clinical Aspects. I. K. Cohen, R. F. Diegelmann and W. J. Lindblad (Eds), pp. 523-540. Philadelphia: Saunders.

Clark, R. A. F. 1988. Overview and general considerations of wound repair. In The Molecular and Cellular Biology of Wound Repair. R. A. F. Clark and P. M. Henson (Eds), pp. 3-34. New York: Plenum.

Clark, R. A. F. 1991. Growth factors and wound repair. J. Cell. Biochem. 46, 1-2.

Clark, R. A. F. 1993. Regulation of fibroplasia in cutaneous wound repair. Am. J. Med. Sci. 306, 42-48.

Dale, P. D., P. K. Maini and J. A. Sherratt. 1994. Mathematical modelling of corneal epithelial wound healing. Math. Biosci. 124, 127-147.

Ehrlich, H. P., A. Desmoulière, R. F. Diegelmann, I. K. Cohen, C. C. Compton, W. L. Garner, Y. Kapanci and G. Gabbiani. 1994. Morphological and immunochemical differences between keloid and hypertrophic scar. Am. J. Pathol. 145, 105-113.

Flint, M. H. 1990. Connective tissue biology. In Dupuytren's Disease: Biology and Treatment. R. M. McFarlane, D. A. McGrouther and M. H. Flint (Eds), p. 13-24. Edinburgh: Churchill Livingstone.

Gabbiani, G. 1992. The biology of the myofibroblast. Kidney Int. 41, 530-532.

Jennings, R. W. and T. K. Hunt. 1992. Overview of postnatal wound healing. In Fetal Wound Healing. N. S. Adzick and M. T. Longaker (Eds), pp. 25-52. New York: Elsevier.

Kirsner, R. S. and W. H. Eaglstein. 1993. The wound healing process. Dermatol. Clin. 11, 629-640.

Martin, P., J. Hopkinson-Woolley and J. McCluskey. 1992. Growth factors and cutaneous wound repair. Prog. Growth Fact. Res. 4, 24-44.

Mast, B. A. 1992. The skin. In Wound Healing: Biochemical and Clinical Aspects. I. K. Cohen, R. F. Diegelmann and W. J. Lindblad (Eds), pp. 344-355. Philadelphia: Saunders.

McCann, B. G., A. Logan, H. Belcher, A. Warn and R. M. Warn. 1993. The presence of myofibroblasts in patients with Dupuytren's Contracture. A possible source for recurrence. J. Hand Surg. Br. 18, 656-661.

Murray, J. C. 1993. Scars and keloids. Dermatol. Clin. 11, 697-708.

Murray, J. C. and S. R. Pinnell. 1992. Keloids and excessive dermal scarring. In Wound Healing: Biochemical and Clinical Aspects. I. K. Cohen, R. F. Diegelmann and W. J. Lindblad (Eds), pp. 500-509. Philadelphia: Saunders. 
Murray, J. D. 1989. Mathematical Biology. New York: Springer-Verlag.

Murray, J. D., P. K. Maini and R. T. Tranquillo. 1988. Mechanochemical models for generating biological pattern and form in development. Phys. Rep. 171, 59-84.

Olsen, L., J. A. Sherratt and P. K. Maini. 1995. A mechanochemical model for adult dermal wound contraction and the permanence of the contracted tissue displacement profile. J. theor. Biol. 177, 113-128.

Raines, E. W., D. F. Bowen-Pope and R. Ross. 1990. Platelet-derived growth factor. In Handbook of Experimental Pharmacology. M. B. Sporn and A. B. Roberts (Eds), Vol. 95, Part I, pp. 173-262. Heidelberg: Springer-Verlag.

Rudolph, R., J. Vande Berg and H. P. Ehrlich. 1992. Wound contraction and scar contracture. In Wound Healing: Biochemical and Clinical Aspects. I. K. Cohen, R. F. Diegelmann and W. J. Lindblad (Eds), pp. 96-114. Philadelphia: Saunders.

Rudolph, R. and J. Vande Berg. 1991. The myofibroblast in Dupuytren's contracture. Hand Clin. 7, 683-692.

Schürch, W., O. Skalli and G. Gabbiani. 1990. Cellular biology. In Dupuytren's Disease: Biology and Treatment. R. M. McFarlane, D. A. McGrouther and M. H. Flint (Eds), pp. 31-47. Edinburgh: Churchill Livingstone.

Sherratt, J. A., E. H. Sage and J. D. Murray. 1993. Chemical control of eukaryotic cell movement: a new model. J. theor. Biol. 162, 23-40.

Skalli, O. and G. Gabbiani. 1988. The biology of the myofibroblast. Rclationship to wound contraction and fibrocontractive diseases. In The Molecular and Cellular Biology of Wound Repair. R. A. F. Clark and P. M. Henson (Eds), pp. 373-402. New York: Plenum.

Skalli, O., W. Schürch, T. Seemayer, R. Lagacé, D. Montandon, B. Pittet and G. Gabbiani. 1989. Myofibroblasts from diverse pathologic settings are heterogeneous in their content of actin isoforms and intermediate filament proteins. Lab. Invest. 60, 275-285.

Sprugel, K. H., J. M. McPherson, A. W. Clowes and R. Ross. 1987. Effects of growth factors in vivo. Am. J. Pathol. 129, 601-613.

Tranquillo, R. T. and J. D. Murray. 1992. Continuum model of fibroblast-driven wound contraction: inflammation-mediation. J. theor. Biol. 158, 135-172.

Traqui, P., D. E. Woodward, G. C. Cruywagen, J. Cook and J. D. Murray. 1996. A mechanical model for fibroblast-driven wound healing. J. Biol. Systems, in press.

Vande Berg, J. S., R. Rudolph, W. L. Poolman and D. R. Disharoon. 1988. Comparative growth dynamics and actin concentration between cultured human myofibroblasts from granulating wounds and dermal fibroblasts from normal skin. Lab. Invest. 61, 532-538. 